\title{
Diffractive deep inelastic scattering in an AdS/CFT inspired model: A phenomenological study
}

\author{
M.A. Betemps ${ }^{1,2}$, V.P. Gonçalves ${ }^{2}$, J. T. de Santana Amaral ${ }^{2}$ \\ 1 Conjunto Agrotécnico Visconde da Graça (CAVG) \\ Universidade Federal de Pelotas, \\ Av. Ildefonso Simões Lopes, 2791 \\ CEP 96060-290, Pelotas, RS, Brazil \\ 2 High and Medium Energy Group (GAME), \\ Instituto de Física e Matemática, \\ Universidade Federal de Pelotas, Caixa Postal 354, \\ CEP 96010-900, Pelotas, RS, Brazil
}

\begin{abstract}
The analytical treatment of the nonperturbative QCD dynamics is one of main open questions of the strong interactions. Currently, it is only possible to get some qualitative information about this regime considering other QCD-like theories, as for example the $N=4$ super Yang-Mills (SYM), where one can perform calculations in the nonperturbative limit of large 't Hooft coupling using the Anti-de Sitter space/Conformal field theory (AdS/CFT). Recently, the high energy scattering amplitude was calculated in the AdS/CFT approach, applied to deep inelastic scattering (DIS) and confronted with the $F_{2}$ HERA data. In this work we extend the nonperturbative AdS/CFT inspired model for diffractive processes and compare its predictions with a perturbative approach based on the Balitsky - Kovchegov (BK) equation. We demonstrate that the AdS/CFT inspired model is not able to describe the current $F_{2}^{D(3)}$ HERA data and predicts a similar behavior to that from BK equation in the range $10^{-7} \lesssim x_{\mathbb{P}} \lesssim 10^{-4}$. At smaller values of $x_{\mathbb{P}}$ the diffractive structure function is predicted to be energy independent.

PACS numbers: $12.38 . \mathrm{Aw}, 13.60 . \mathrm{Hb}, 11.25 . \mathrm{Sq}$
\end{abstract}

\section{INTRODUCTION}

The physics of high density QCD has become an increasingly active subject of research, both from experimental and theoretical points of view (For recent reviews see [1]). In deep inelastic scattering (DIS) the high parton density regime corresponds to the small $x$ region and represents the challenge of studying the interface between the perturbative and nonperturbative QCD, with the peculiar feature that this transition is taken in a kinematical region where the strong coupling constant $\alpha_{s}$ is small. By the domain of perturbative QCD we mean the region where the parton picture has been developed and the separation between the short and long distance contributions (the collinear factorization) is made possible by the use of the operator product expansion (OPE). The Dokshitzer-Gribov-Lipatov-Altarelli-Parisi (DGLAP) equations [2], which resums terms of the type $\alpha_{s}^{n} \ln ^{n} Q^{2}$, are the evolution equations in this kinematical region. These equations are valid at leading twist, i.e. at a large value of the photon virtuality $Q^{2}$, where a subclass of all possible Feynmann graphs are dominant and the hard coefficient function is connected to the proton by only two parton lines (For more details see e.g. Ref. [3]). For small values of $Q^{2}$, this picture has corrections predicted by the OPE that contribute at relative order $\mathcal{O}\left(1 / Q^{2}\right)$ and beyond $\left[\mathcal{O}\left(\frac{1}{Q^{2}}\right)^{n}, n=2,3, \ldots\right]$. These are commonly called higher twist corrections.

In the limit of small values of $x$, terms of the type $\alpha_{s}^{n} \ln ^{n} 1 / x$ become relevant and the DGLAP equation is not expected to be valid. In this kinematical region one expects to see new features inside the proton: the density of gluons and quarks becomes very high and an associated new dynamical effect is expected to stop the further growth of the structure functions. In particular, for a fixed hard scale $Q^{2} \gg \Lambda_{Q C D}^{2}$, the OPE eventually breaks down at sufficiently small $x$ [4]. Ultimately, the physics in the region of high parton densities will be described by nonperturbative methods, but this is still waiting for a satisfactory solution in QCD. However, the transition from the moderate $x$ region towards the small $x$ limit may possibly be accessible in perturbation theory, and, hence, allows us to test the ideas about the onset of nonperturbative dynamics. Currently, this transition is quite well described by the Color Glass Condensate (CGC) formalism, whose central result is the Jalilian-Marian-Iancu-McLerran-Weigert-Leonidov-Kovner (JIMWLK) equation [5-12]. When applied to the scattering between a simple projectile and a CGC, one obtains an infinite hierarchy of coupled equations for the correlators of Wilson lines, the Balitsky-JIMWLK (B-JIMWLK) hierarchy 
[13 17]. In the mean field approximation, this hierarchy reduces to a single closed nonlinear equation, the BalitskyKovchegov (BK) equation [13, 18, 19], which describes the evolution with energy of the dipole-hadron scattering amplitude in the large $N_{c}$ limit $\left(N_{c}\right.$ is the number of colors). The CGC physics is characterized by a saturation scale $Q_{s}$ which is related to the critical transverse size for the unitarization of the scattering amplitudes and is predicted by the BK equation to be an increasing function of the energy and atomic number dependent $\left[Q_{s}^{2}=Q_{0}^{2}\left(\frac{x_{0}}{x}\right)^{\lambda} A^{\alpha}\right.$ with $\lambda$ and $\alpha>0$ ]. Currently, the analysis of HERA and RHIC data using phenomenological models based on CGC physics implies that $Q_{s} \approx 1-3 \mathrm{GeV}$ for proton and nuclei [1]. As this scale is not very large at these machines, contributions from nonperturbative dynamics cannot be completely disregarded before estimating its magnitude.

The analytical treatment of the nonperturbative QCD dynamics is one of main open questions of the strong interactions. Currently, it is only possible to get some qualitative information about this regime considering other QCD-like theories, which are accessible at strong coupling and share some of the basic features of QCD at high energy. One such theory is the $N=4$ super Yang-Mills (SYM) where one can perform calculations in the nonperturbative limit of large 't Hooft coupling using the Anti-de Sitter space/Conformal field theory (AdS/CFT) correspondence 20 22] (For a recent review see 23] ). However, it should be emphasized that QCD is a confining theory with a dynamically generated scale $\Lambda \approx 200 \mathrm{MeV}$, whereas $N=4 \mathrm{SYM}$ is a conformal theory with no scales and thus does not have confinement. Moreover, in $N=4 \mathrm{SYM}$ there is no mass gap in the asymptotic particle spectrum, which implies that the Froissart bound is not expected to be valid. A correspondence closer to QCD can be obtained if the conformal invariance of the theory is broken. The simplest way to break conformal invariance is by introducing a hard cut-off in AdS space which can be interpreted as an infrared scale of the gauge theory. Another possibility is to break conformal invariance softly through a background dilaton field. These approaches have been used in last years by several authors in order to study the DIS and calculate the high energy scattering amplitudes in the AdS/CFT formalism [24 32]. In general, these authors treat the DIS off a shockwave considering the interaction of a virtual photon with the target. In contrast, in [33], DIS with a nucleus target was considered in the framework of the shockwave approximation with the Wilson loop immersed into a gauge field background calculated following the proposal by Janik and Peschanski [34]. These authors have considered that the projectile is a color dipole, as represented by a Nambu-Goto string. This model was confronted with the available HERA data in Ref. [35]. The basic idea was to construct a dipole-target cross section inspired in the AdS/CFT approach valid at small values of the photon virtuality $Q^{2}$, which can be viewed as complementary to the perturbative descriptions based on CGC physics. A surprising prediction of this AdS/CFT inspired model is the $x$ independence of the $F_{2}$ structure function at very small $x$ and $Q^{2}$ in a region where there is no experimental data yet, which is directly associated to the behavior of the saturation scale at high energies. This result can be contrasted with the prediction of the high density QCD approaches, where $F_{2} \propto \ln (1 / x)$ in the asymptotic regime [36]. Although the relevance of the AdS/CFT results for our real QCD world is far from being clear, the fact that this model is able to describe the $F_{2}$ HERA data in the kinematical region of $x<6 \times 10^{-5}$ and $Q^{2}<2.5 \mathrm{GeV}^{2}$ [35] motivates one to check what are the predictions of this model for other observables. In particular, it is very important to determine the signatures of the AdS/CFT inspired model in order to discriminate unambiguously from other approaches, as for example the CGC formalism.

Recently, the running coupling corrections to BK equation were calculated through the ressumation of $\alpha_{s} N_{f}$ contributions ( $N_{f}$ being the number of flavors) to all orders, allowing the estimation of the soft gluon emission and running coupling corrections to the evolution kernel [37-40]. The solution of this improved BK equation was studied in detail in Refs. 38, 41]. More recently, a global analysis of the small $x\left(x \leq 10^{-2}\right)$ data for the proton structure function in the kinematical range $0.045 \mathrm{GeV}^{2} \leq Q^{2} \leq 800 \mathrm{GeV}^{2}$ using the improved BK equation was performed [42] (See also Ref. [43]). In contrast to the BK equation at leading order (LO), which fails to describe data, the inclusion of running coupling effects to evolution renders BK equation compatible with them. In particular, the $F_{2}$ data in the region of very small $Q^{2}$ is also quite well described by the improved BK equation. It implies that the current $F_{2}$ data at low $Q^{2}$ and $x$ is not able to discriminate between the nonperturbative AdS/CFT inspired model and the perturbative CGC physics.

In this paper we analyse the predictions of the AdS/CFT inspired model for diffractive processes and compare its results with those from the running coupling Balitsky-Kovchegov (RC BK) solution obtained in Ref. 42]. Our main motivation comes from the fact that in diffractive deep inelastic scattering (DDIS), mainly on $F_{2}^{D}$, the interplay between hard and soft regimes is more explicit [44, 45]. Basically, the partonic fluctuations of the virtual photon can lead to configurations of different sizes when analysed in the proton rest frame. The study of the diffractive dissociation of protons has shown that for real photons $\left(Q^{2} \approx 0\right)$, where the transverse size of the incoming pair is approximately that of a hadron, the energy dependence is compatible with the expectations based on the soft Pomeron exchange. On the other hand, at large $Q^{2}$ the energy dependence is higher than that of the soft Pomeron, suggesting that pQCD effects may become visible for small incoming quark-antiquark pairs. Therefore, as the $F_{2}^{D}$ structure function is inclusive to the hard and soft contributions to the dynamics, its study can be useful to discriminate between the RC BK and AdS/CFT predictions.

This paper is organized as follows. In Section II we present the dipole formalism, which describes the inclusive and 
diffractive deep inelastic scattering in a common approach. Within this formalism, we present the parameterization for the scattering amplitude inspired in the AdS/CFT correspondence proposed in [33], which has been recently used to describe the HERA data for the $F_{2}$ proton structure function [35]. Moreover, we analyze the $\boldsymbol{r}$ and $x$ - dependence of the scattering amplitude predicted by the AdS/CFT inpired model and compare it with the RC BK solution. In Section III we analyze the predictions of these models for the inclusive and diffractive overlap functions and calculate the diffractive structure function for small $Q^{2}$. For completeness, the $F_{2}$ structure function is also calculated for similar values of $Q^{2}$. Finally, we calculate the ratio between the diffractive and inclusive total cross sections, $R_{\sigma}=\sigma_{\text {tot }}^{D} / \sigma_{\text {tot }}$, and study its energy dependence considering the AdS/CFT inspired model and the RC BK solution. The conclusions are presented in Section IV.

\section{DIPOLE FORMALISM}

In the study of the observables of lepton-hadron deep inelastic scattering (DIS) at small $x$, it is convenient to see the scattering between the virtual photon (which is exchanged in the lepton-hadron interaction) and the hadron in the dipole frame: in this frame, most of the total energy is carried by the hadron, but the virtual photon has enough energy to dissociate into a quark-antiquark $(q \bar{q})$ pair, a dipole, before the scattering. In such special frame, the QCD description of DIS at small $x$ can be interpreted as a two-step process [46]: the virtual photon $\gamma^{*}$ splits into the $q \bar{q}$ dipole, with transverse separation $\boldsymbol{r}$, which subsequently interacts with the hadron $h$. In terms of cross sections for the transversely $(T)$ and longitudinally $(L)$ polarized photons, the $F_{2}$ structure function is given by $F_{2}\left(x, Q^{2}\right)=\frac{Q^{2}}{4 \pi^{2} \alpha_{e m}} \sigma_{t o t}$, where $\alpha_{e m}$ is the electromagnetic coupling constant and $\sigma_{t o t}$ is the total (inclusive) $\gamma^{*} h$ cross section expressed by

$$
\sigma_{t o t}=\sum_{i=T, L} \int d^{2} \boldsymbol{r} d z\left|\Psi_{i}\left(\boldsymbol{r}, z, Q^{2}\right)\right|^{2} \sigma_{d i p}(x, \boldsymbol{r})
$$

The functions $\Psi_{T, L}$ are the light-cone wave functions of the virtual photon, $z$ is the photon momentum fraction carried by the quark (for details see e.g. Ref. [47]) and $\sigma_{\text {dip }}$ is the dipole-hadron cross section.

Diffractive processes in deep-inelastic scattering (DDIS) are characterized by the presence of large rapidity gaps in the hadronic final state and are associated to a Pomeron exchange. Within the framework of the perturbative QCD (pQCD), the Pomeron is associated with the resummation of leading logarithms in $s$ (center of mass energy squared) and at lowest order is described by the two-gluon exchange. These processes are of particular interest, because the hard photon in the initial state gives rise to the hope that, at least in part, the scattering amplitude can be calculated in pQCD. In the dipole approach the total diffractive cross sections take the following form (See e.g. Refs. [46-48]),

$$
\sigma_{T, L}^{D}=\left.\int_{-\infty}^{0} d t e^{B_{D} t} \frac{d \sigma_{T, L}^{D}}{d t}\right|_{t=0}=\left.\frac{1}{B_{D}} \frac{d \sigma_{T, L}^{D}}{d t}\right|_{t=0}
$$

where

$$
\left.\frac{d \sigma_{T, L}^{D}}{d t}\right|_{t=0}=\frac{1}{16 \pi} \int d^{2} \mathbf{r} \int_{0}^{1} d \alpha\left|\Psi_{T, L}(\alpha, \mathbf{r})\right|^{2} \sigma_{d i p}^{2}(x, \boldsymbol{r}),
$$

and it is assumed a factorizable dependence on momentum transfer $t$, on which the dependence is given through an exponential with diffractive slope $B_{D}$. The diffractive processes can be analyzed in more detail by studying the behavior of the diffractive structure function $F_{2}^{D(3)}\left(Q^{2}, \beta, x_{\mathbb{P}}\right)$. Following Ref. [49] we assume that the diffractive structure function is given by

$$
F_{2}^{D(3)}\left(Q^{2}, \beta, x_{\mathbb{P}}\right)=F_{q \bar{q}, L}^{D}+F_{q \bar{q}, T}^{D}+F_{q \bar{q} g, T}^{D},
$$

where the $q \bar{q} g$ contribution with longitudinal polarization is not present because it has no leading logarithm in $Q^{2}$. The different contributions can be calculated and for the $q \bar{q}$ contributions they read [46, 49, 50]

$$
\begin{gathered}
x_{\mathbb{P}} F_{q \bar{q}, L}^{D}\left(Q^{2}, \beta, x_{\mathbb{P}}\right)=\frac{3 Q^{6}}{32 \pi^{4} \beta B_{D}} \sum_{f} e_{f}^{2} 2 \int_{\alpha_{0}}^{1 / 2} d \alpha \alpha^{3}(1-\alpha)^{3} \Phi_{0}, \\
x_{\mathbb{P}} F_{q \bar{q}, T}^{D}\left(Q^{2}, \beta, x_{\mathbb{P}}\right)=\frac{3 Q^{4}}{128 \pi^{4} \beta B_{D}} \sum_{f} e_{f}^{2} 2 \int_{\alpha_{0}}^{1 / 2} d \alpha \alpha(1-\alpha)\left\{\epsilon^{2}\left[\alpha^{2}+(1-\alpha)^{2}\right] \Phi_{1}+m_{f}^{2} \Phi_{0}\right\}
\end{gathered}
$$



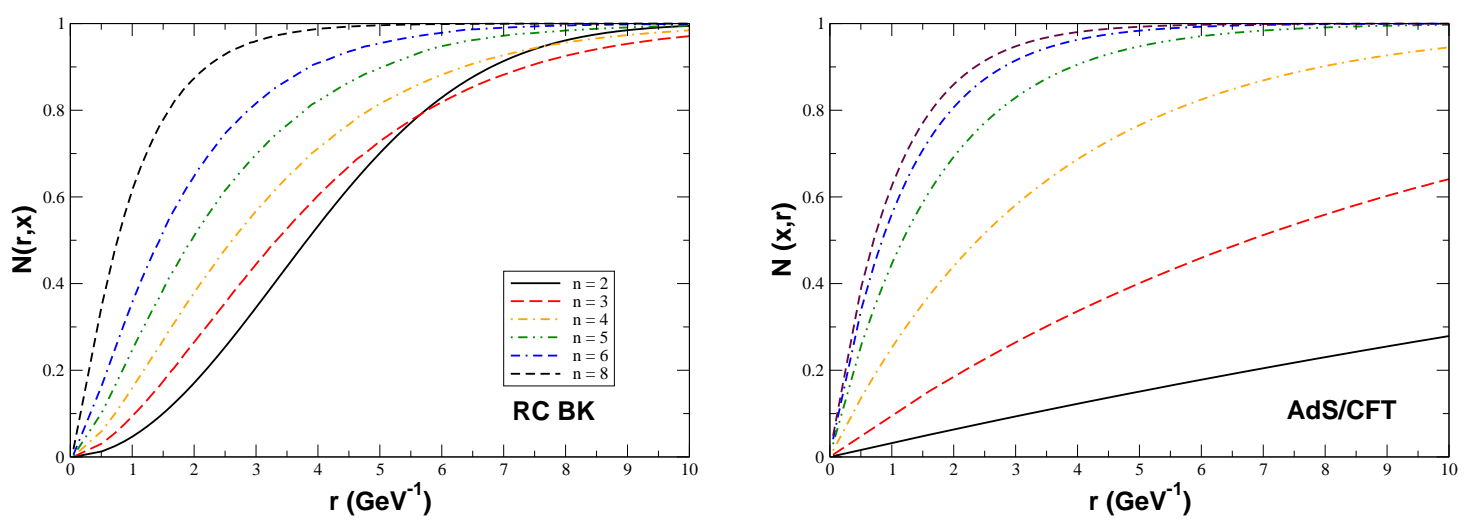

FIG. 1: Dependence of the dipole scattering amplitude in the pair separation $r$ at different values of $x\left(x=10^{-n}\right)$.

where the lower limit of the integral over $\alpha$ is given by $\alpha_{0}=\frac{1}{2}\left(1-\sqrt{1-\frac{4 m_{f}^{2}}{M_{X}^{2}}}\right)$, the sum is performed over the quark flavors and [51]

$$
\Phi_{0,1} \equiv\left(\int_{0}^{\infty} r d r K_{0,1}(\epsilon r) \sigma_{d i p}\left(x_{\mathbb{P}}, \boldsymbol{r}\right) J_{0,1}(k r)\right)^{2}
$$

The $q \bar{q} g$ contribution, within the dipole picture at leading $\ln Q^{2}$ accuracy, is given by [49, 50, 52, 53]

$$
\begin{aligned}
& x_{\mathbb{P}} F_{q \bar{q} g, T}^{D}\left(Q^{2}, \beta, x_{\mathbb{P}}\right)=\frac{81 \beta \alpha_{S}}{512 \pi^{5} B_{D}} \sum_{f} e_{f}^{2} \int_{\beta}^{1} \frac{\mathrm{d} z}{(1-z)^{3}}\left[\left(1-\frac{\beta}{z}\right)^{2}+\left(\frac{\beta}{z}\right)^{2}\right] \\
& \times \int_{0}^{(1-z) Q^{2}} \mathrm{~d} k_{t}^{2} \ln \left(\frac{(1-z) Q^{2}}{k_{t}^{2}}\right)\left[\int_{0}^{\infty} u \mathrm{~d} u \sigma_{d i p}\left(u / k_{t}, x_{\mathbb{P}}\right) K_{2}\left(\sqrt{\frac{z}{1-z} u^{2}}\right) J_{2}(u)\right]^{2} .
\end{aligned}
$$

As pointed in Ref. [54], at small $\beta$ and low $Q^{2}$, the leading $\ln (1 / \beta)$ terms should be resumed and the above expression should be modified. However, as a description with the same quality using the Eq. (8) is possible by adjusting the coupling [54], in what follows we will use this expression for our phenomenological studies. We use the standard notation for the variables $\beta=Q^{2} /\left(M_{X}^{2}+Q^{2}\right), x_{\mathbb{P}}=\left(M_{X}^{2}+Q^{2}\right) /\left(W^{2}+Q^{2}\right)$ and $x=Q^{2} /\left(W^{2}+Q^{2}\right)=\beta x_{\mathbb{P}}$, where $M_{X}$ is the invariant mass of the diffractive system, $B_{D}$ is the diffractive slope and $W$ the total energy of the $\gamma^{*} p$ system.

The main input for the calculations of inclusive and diffractive observables in the dipole picture is $\sigma_{d i p}(x, \boldsymbol{r})$ which is determined by the QCD dynamics at small $x$. In the eikonal approximation, it is given by:

$$
\sigma_{d i p}(x, \boldsymbol{r})=2 \int d^{2} \boldsymbol{b} \mathcal{N}(x, \boldsymbol{r}, \boldsymbol{b})
$$

where $\mathcal{N}(x, \boldsymbol{r}, \boldsymbol{b})$ is the forward scattering amplitude for a dipole with size $r=|\boldsymbol{r}|$ and impact parameter $\boldsymbol{b}$ which can be related to expectation value of a Wilson loop [1]. It encodes all the information about the hadronic scattering, and thus about the non-linear and quantum effects in the hadron wave function. In general, it is assumed that the impact parameter dependence of $\mathcal{N}$ can be factorized as $\mathcal{N}(x, \boldsymbol{r}, \boldsymbol{b})=\mathcal{N}(x, \boldsymbol{r}) S(\boldsymbol{b})$, where $S(\boldsymbol{b})$ is the profile function in impact parameter space, which implies $\sigma_{\text {dip }}(x, \boldsymbol{r})=\sigma_{0} \mathcal{N}(x, \boldsymbol{r})$. The forward scattering amplitude $\mathcal{N}(x, \boldsymbol{r})$ can be obtained by solving the BK evolution equation 42] or considering phenomenological QCD inspired models to describe the interaction of the dipole with the target [35, 48, 49, 54 [69]. BK equation is the simplest nonlinear evolution equation for the dipole-hadron scattering amplitude, being actually a mean field version of the first equation of the B-JIMWLK hierarchy [13, 14]. In its linear version, it corresponds to the Balitsky-Fadin-Kuraev-Lipatov (BFKL) equation [70]. The LO BK equation presents some difficulties when applied to study DIS small- $x$ data, in particular, some studies concerning this equation [71 75] have shown that the resulting saturation scale grows much faster with increasing energy $\left(Q_{s}^{2} \sim x^{-\lambda}\right.$, with $\left.\lambda \approx 0.5\right)$ than that extracted from phenomenology $(\lambda \sim 0.2-0.3)$. This difficulty could be solved by considering higher order corrections to LO BK equation, which were recently calculated 37 40] and have shown to successfully describe small- $x\left(x \leq 10^{-2}\right)$ data for the proton structure function [42]. In what follows we will use in our RC BK calculations the public-use code available in [76]. 

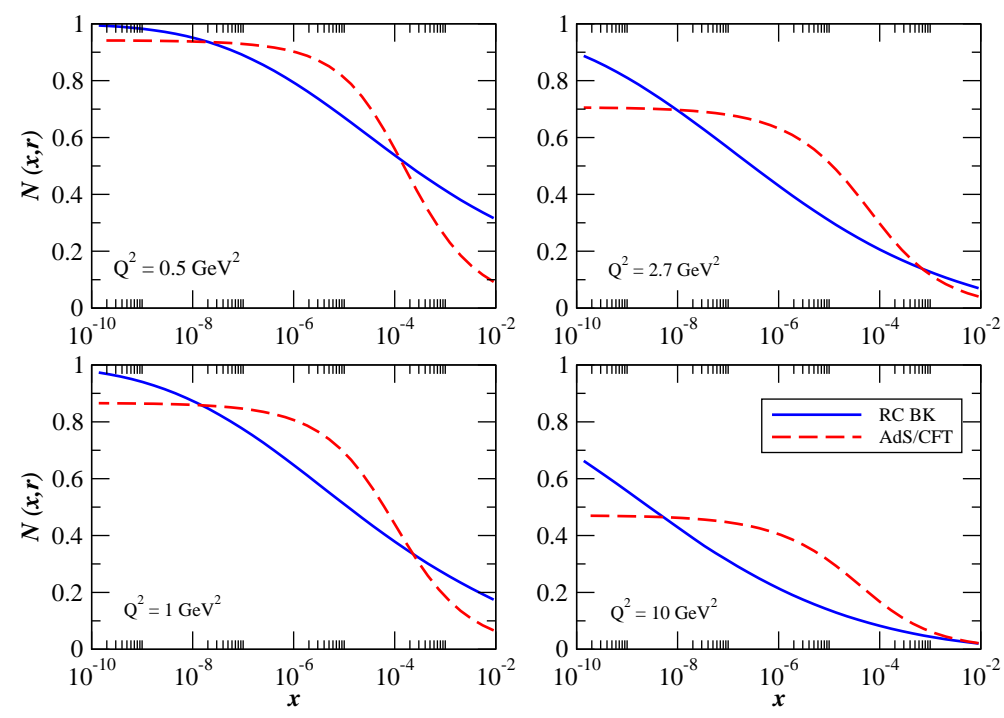

FIG. 2: Energy dependence of the scattering amplitude as a function of $x$ at different values of $Q^{2}\left(r^{2}=1 / Q^{2}\right)$.

An alternative approach is to calculate the scattering amplitude at high energy for a strongly coupled $N=4$ super Yang-Mills theory using the AdS/CFT correspondence. In [33] the authors have modelled the nucleus by a metric of a shock wave in $\mathrm{AdS}_{5}$ and calculated the total cross section or DIS on a large nucleus. The expectation value of the Wilson loop, which is directly related to $\mathcal{N}$, was calculated by finding the extrema of the Nambu-Goto action for an open string attached to the quark and antiquark lines of the loop in the background of an $\mathrm{AdS}_{5}$ shock wave. The resulting scattering amplitude can be expressed in terms of a AdS/CFT saturation scale, which is energy independent at very small $x$ and depends very strongly on the atomic number of the nucleus $\left(\propto A^{1 / 3}\right)$. In [35] it was used to describe the HERA data for $F_{2}$ proton structure function in the small $\left(x, Q^{2}\right)$ range and a parametrization for the dipole-proton scattering amplitude was provided. In this AdS/CFT inspired model the scattering amplitude is given by [33]

$$
\mathcal{N}(r, x)=1-\exp \left[-\frac{\mathcal{A}_{0} x r}{\mathcal{M}_{0}^{2}(1-x) \pi \sqrt{2}}\left(\frac{1}{\rho_{m}^{3}}+\frac{2}{\rho_{m}}-2 \mathcal{M}_{0} \sqrt{\frac{1-x}{x}}\right)\right]
$$

with

$$
\begin{aligned}
\rho_{m} & =\left\{\begin{array}{ll}
\left(\frac{1}{3 m}\right)^{1 / 4} \sqrt{2 \cos \left(\frac{\theta}{3}\right)} & : m \leq \frac{4}{27} \\
\sqrt{\frac{1}{3 m \Delta}+\Delta} & : m>\frac{4}{27}
\end{array},\right. \\
\Delta & =\left[\frac{1}{2 m}-\sqrt{\frac{1}{4 m^{2}}-\frac{1}{27 m^{3}}}\right]^{1 / 3} \\
m & =\frac{\mathcal{M}_{0}^{4}(1-x)^{2}}{x^{2}}, \\
\cos (\theta) & =\sqrt{\frac{27 m}{4}} .
\end{aligned}
$$

In the above equations, $\mathcal{M}_{0}=b_{0} c_{0}$, where $b_{0}$ relates the virtuality of the photon to the dipole size, $b_{0}=r Q$, and $c_{0}=\Gamma^{2}\left(\frac{1}{4}\right) /(2 \pi)^{3 / 2} \approx 0.83$; this is the value predicted by AdS/CFT calculations, however, from the fit to HERA data it assumes a value about 2 orders of magnitude smaller, which may be explained by the fact that $N=4 \mathrm{SYM}$ theory and QCD are diferent from each other (see the discussion in Section IV of Ref.[35]). $\mathcal{A}_{0}=\sqrt{\lambda_{\mathrm{YM}}} \Lambda$, where $\lambda_{\mathrm{YM}}=g_{\mathrm{YM}}^{2} N_{c}$ denotes the 't Hooft coupling with $g_{\mathrm{YM}}$ the Yang-Mills coupling constant, and $\Lambda$ is chosen to be $\Lambda=1$ $\mathrm{GeV}$. The resulting saturation scale has the following form:

$$
Q_{s}^{\mathrm{AdS}}(x)=\frac{2 \mathcal{A}_{0} x}{\mathcal{M}_{0}^{2}(1-x) \pi}\left(\frac{1}{\rho_{m}^{3}}+\frac{2}{\rho_{m}}-2 \mathcal{M}_{0} \sqrt{\frac{1-x}{x}}\right) .
$$



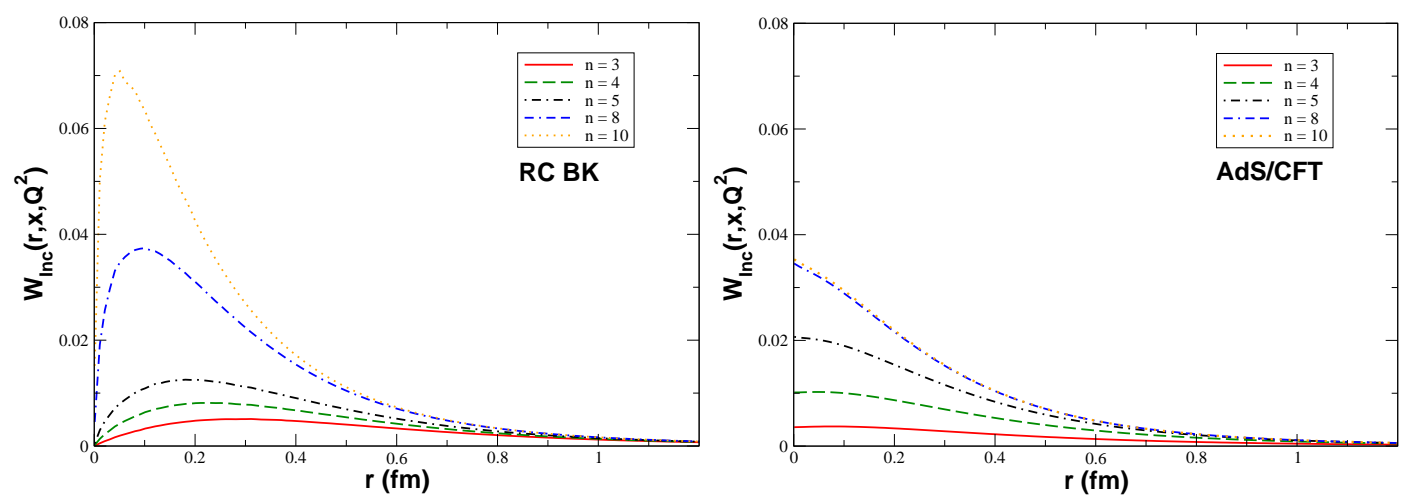

FIG. 3: Pair separation dependence of the function $W_{\text {Inc }}\left(r, x, Q^{2}\right)$ for distinct values of $x\left(x=10^{-n}\right)$ and $Q^{2}=1 \mathrm{GeV}^{2}$.

It has its values in the interval $1 \div 3 \mathrm{GeV}$ in the range $6.2 \times 10^{-7} \leq x \leq 6 \times 10^{-5}$ and has a particular property: for extremely small values of $x$, the saturation scale saturates, which is in sharp contrast with its usual form in QCD, where it grows with decreasing $x$. This behavior implies that the DIS cross sections will present a slow growth with the energy, similar to that predicted by soft pomeron models [33]. In what follows we use $m_{f}=140 \mathrm{MeV}$ for the (light) quark masses and the other parameters are chosen based on the analysis done in [35]; in particular, the value of the t'Hooft coupling constant is chosen to be $\lambda_{\mathrm{YM}}=20$ : this choice is consistent with the fact that AdS/CFT correspondence is valid for $\lambda_{\mathrm{YM}} \gg 1$ and motivated mainly because, besides the resulting good $\chi^{2}$, the results of fit to HERA data change only a little bit for a wide range $\lambda_{\mathrm{YM}} \geq 20$. This gives $\mathcal{M}_{0}=6.54 \times 10^{-3}$ and $\sigma_{0}=22.47 \mathrm{mb}$.

In Fig. 1 we show the pair separation dependence of the scattering amplitude $\mathcal{N}$ for different values of $x$ considering the AdS/CFT inspired model and the RC BK solution (For a related discussion see Refs. 77, 78]). Although the AdS/CFT model is constructed in order to describe small $x\left(\leq 10^{-4}\right)$ we also present its predictions at larger $x$ in order to compare with the RC BK solution. The first aspect that can be observed is the large difference between the predictions at $x>10^{-4}$. While the RC BK solution predicts that the scattering amplitude saturates at large $x$, the AdS/CFT model still predicts a growth in the $r$-range considered. In the range $10^{-6} \leq x \leq 10^{-4}$ the behavior predicted by the two models for $\mathcal{N}$ is similar, which is expected since it is the range of the HERA data used to constrain the main parameters of the AdS/CFT model. In this region both models predict that $\mathcal{N}$ saturates at large pair separation. At smaller values of $x$, the two models predict different behaviors. While the scattering amplitude in the AdS/CFT model is almost $x$ independent, the RC BK solution is significantly modified when $x$ goes to smaller values. In particular, at $x \leq 10^{-8}$ the scattering amplitude in the AdS/CFT inspired model becomes energy independent. It can be observed in more detail in Fig. 2, where we analyze the $x$ dependence of the scattering amplitude for different values of $Q^{2}$, i.e. different values of the squared pair separation if we assume $r^{2}=1 / Q^{2}$. As expected from Fig. 1, the two predictions are very distinct at large $x\left(\geq 10^{-4}\right)$ and large pair separations $\left(Q^{2}=0.5\right.$ and $1 \mathrm{GeV}^{2}$ ), being smaller for larger values of $Q^{2}$. In contrast to the RC BK solution, which predicts that $\mathcal{N}$ grows at small $x$ and large $Q^{2}$, the AdS/CFT model predicts that $\mathcal{N}$ saturates at $x \leq 10^{-6}$ independently of the pair separation. As in the dipole approach the observables are determined by the scattering amplitude, we can expect that these differences between the models also be observed in the inclusive and diffractive structure functions.

\section{RESULTS}

In DIS the partonic fluctuations of the virtual photon can lead to configurations of different sizes when analysed in the dipole frame. The size of the configuration will depend on the relative transverse momentum $k_{T}$ of the $q \bar{q}$ pair. The small size configurations are calculated using perturbative QCD and at small Bjorken scaling variable $x$ the smallness of the cross section (color transparency) is compensated by the large gluon distribution. For large size configurations one expects to be in the regime of soft interactions. In the inclusive measurement of final states one sums over both small-distance and large-distance configurations. The contribution of small and large pair separations for inclusive and diffractive observables can be studied considering the corresponding overlap functions which are given by:

$$
W_{\text {Inc }}\left(r, x, Q^{2}\right)=2 \pi r \sum_{i=T, L} \int d z\left|\Psi_{i}\left(z, r, Q^{2}\right)\right|^{2} \sigma_{d i p}(x, r)
$$



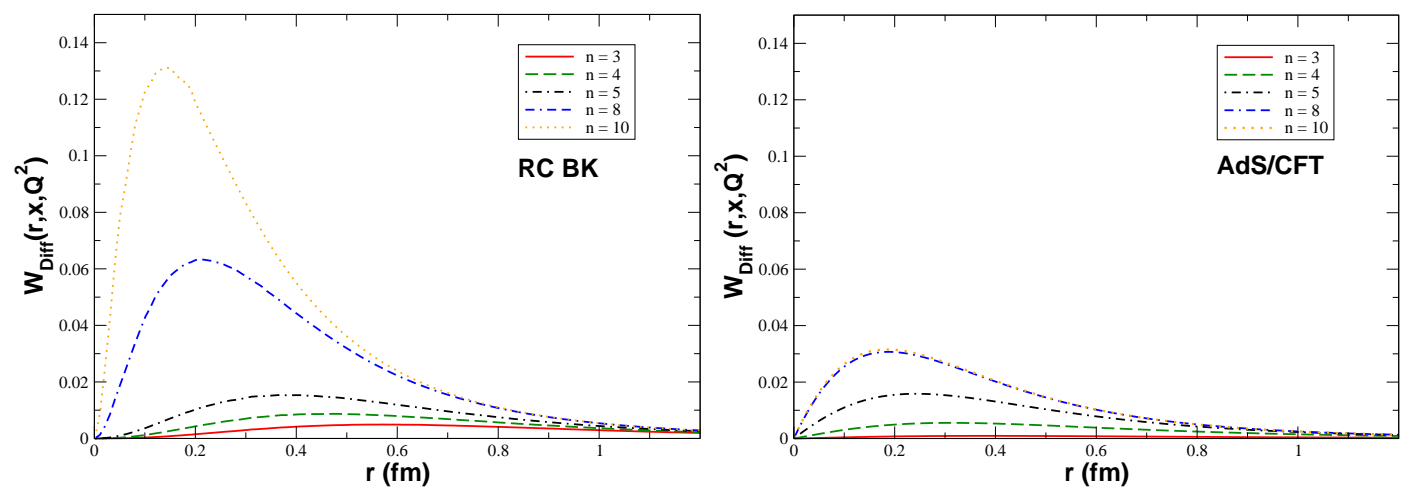

FIG. 4: Pair separation dependence of the function $W_{D i f f}\left(r, x, Q^{2}\right)$ for distinct values of $x\left(x=10^{-n}\right)$ and $Q^{2}=1 \mathrm{GeV}^{2}$.

and

$$
W_{D i f f}\left(r, x, Q^{2}\right)=2 \pi r \sum_{i=T, L} \int d \alpha\left|\Psi_{i}\left(\alpha, r, Q^{2}\right)\right|^{2} \sigma_{d i p}^{2}(x, r)
$$

It is important to emphasize that the behavior of the overlap functions are strongly dependent on the scattering amplitude. In particular, as shown in [4], if one considers a model where $\mathcal{N} \propto r^{2}$ (linear dynamics) the diffractive overlap function is dominated by large pair separations and consequently the diffractive cross sections are determined by nonperturbative physics. Moreover, it is the energy dependence of the scattering amplitudes which determine the $x$ dependence of the overlap functions.

In Fig. 3 we present our results for the inclusive overlap function considering the AdS/CFT inspired model and the RC BK solution at different values of $x\left(x=10^{-n}\right.$ with $\left.n=3,4,5,8,10\right)$ and $Q^{2}=1 \mathrm{GeV}^{2}$. We have that both models predict a similar behavior for the large $r$ region, which implies that the main contribution for inclusive observables comes from small pair separations. As $r \rightarrow 0$ the RC BK solution predicts that $W_{\text {Inc }}$ goes to zero, which is directly associated to the BFKL behavior present in the BK equation at very small $r$. In contrast, the AdS/CFT inspired model predicts that in this limit $W_{I n c}$ goes to a constant value, which is energy-dependent. Another aspect which should be emphasized is that the inclusive overlap becomes energy independent at $x \leq 10^{-8}$ for the AdS/CFT model, in agremment with the behavior of the corresponding scattering amplitude. In contrast, RC BK solution implies that $W_{I n c}$ is energy dependent. These behaviors have direct impact in the predictions for the $F_{2}$ structure function, as will be verified below. Finally, the peak of the inclusive overlap function occurs at smaller values of $r$ with the decreasing of $x$.

As discussed in the Introduction, the main theoretical interest in diffraction is centered around the interplay between the soft and hard physics. The ability to clearly separate the regimes dominated by soft and hard processes is essential in exploring QCD at both quantitative and qualitative levels. Moreover, as the diffractive observables ( $\sigma_{T, L}^{D}$ for example) are proportional to $\sigma_{d i p}^{2}$, these are more sensitive to the large pair separation contributions [49]. These features of the diffractive processes make DDIS an ideal scenario to check the AdS/CFT inspired dipole model, since this is a nonperturbative inspired model of saturation. In Fig. 团we present our results for the diffractive overlap function considering the AdS/CFT inspired model and the RC BK solution at different values of $x\left(x=10^{-n}\right.$ with $n=$ $3,4,5,8,10)$ and $Q^{2}=1 \mathrm{GeV}^{2}$. We can see that both models predict that the large pair separations do not contribute significantly for the diffractive overlap function. Moreover, $W_{D i f f}\left(r, x, Q^{2}\right)$ goes to zero at $r \rightarrow 0$ for both models. The peak of the distribution occurs at smaller values of $r$ with the decreasing $x$. In comparison with the inclusive case, it peaks at larger values of $r$, which implies that the main contribution for diffractive observables comes from a different range of values of pair separation and consequently probe distinct aspects of the QCD dynamics present in the scattering amplitude. The small $x$ effects in the scattering amplitude predicted by the AdS/CFT inspired model and the RC BK solution drive the energy behavior of the diffractive overlap function. In Fig. 2 the $x$-dependence of the $\mathcal{N}(x, r)$ shows that for $x<10^{-6}$ the AdS/CFT predicts a saturation of the amplitude, while the RC BK predicts a saturation only for $x<10^{-10}$. This is one of the reasons for the distinct normalization of the overlap function. The saturation of the AdS/CFT dipole model for small $x$ is more evident if one compares the overlap function for $x=10^{-8}$ and $x=10^{-10}$, since the curves are identical. The AdS/CFT dipole amplitude presents smaller values when 

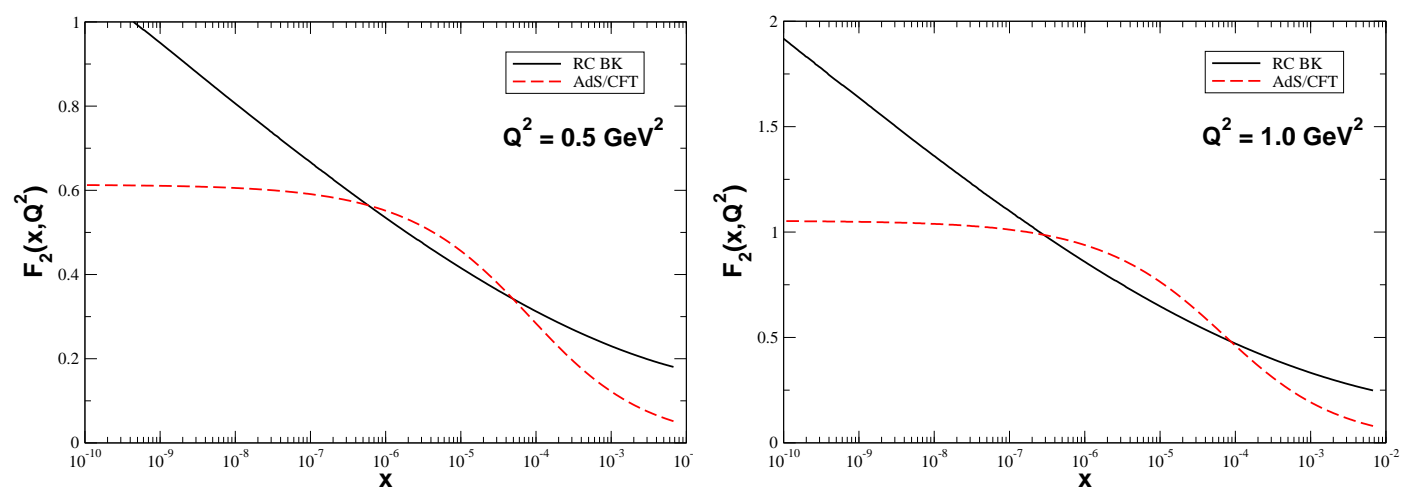

FIG. 5: The $F_{2}$ structure function for two distinct values of $Q^{2}$.

compared to the RC BK model in the region $x \gtrsim 10^{-4}$ and $x \lesssim 10^{-6}$. This explains the different normalizations of the overlap functions in the Fig. 4

Having addressed the main features of the overlap functions, we continue by studying the inclusive and diffractive proton structure functions. We focus our analysis in the small $\left(x, Q^{2}\right)$ kinematical range probed by HERA and which could be tested in future ep colliders at the TeV energy scale, as for example the LHeC project (For a recent discussion see [79]). In Fig. 5 we show the $F_{2}$ proton structure function as a function of $x$ for two different small values of the photon virtuality, $Q^{2}=0.5$ and $1 \mathrm{GeV}^{2}$, using the AdS/CFT inspired model (dashed line) and, for the sake of comparison, the RC BK solution (solid line). One can clearly see that both analyses match only in a restrict region, for values of $x$ between $\sim 10^{-4}$ and $\sim 10^{-7}$; such an interval corresponds to the HERA kinematical regime investigated in Ref.[35]. For values of $x$ larger than $10^{-4}$ and, specially, smaller than $\sim 10^{-7}$ (for which there are no available experimental data) the behaviors of the curves become distinct. This distinct behavior of $F_{2}\left(x, Q^{2}\right)$ is related to the $x$ dependence of the scattering amplitude in the AdS/CFT and RC BK models, analysed in the Fig. 2. In particular, we have that the AdS/CFT inspired model predicts the saturation of the $F_{2}$ structure function at $\lesssim 10^{-6}$. At $x=10^{-8}$ the predictions differ by a factor 1.4 .

Let us now discuss the predictions of the AdS/CFT inspired model for the diffractive structure function. As explained before the main theoretical interest in diffraction is centered around the interplay between the soft and hard physics. In particular, in the inclusive measurement of diffractive final states, where the diffractive structure function is derived, one sums over both small-distance and large-distance configurations. As in the diffractive cross section we integrate over both perturbative and nonperturbative regions of the phase space, there is a competition between these two pieces. Therefore, in principle, this observable is ideal to test the nonperturbative physics present in the AdS/CFT inspired model. Before the comparison of the predictions with the HERA data a comment is in order. The diffractive structure function has been measured by the H1 and ZEUS Collaborations in ep collisions at HERA in a large kinematical $\left(Q^{2}, x\right)$ range. However, as the model proposed in [35] is only valid at small $Q^{2}$ we will select the HERA data in this range. This limits our comparison to the ZEUS data at $Q^{2}=2.7 \mathrm{GeV}^{2}[80$, which is similar to the upper limit considered in [35]. In Fig. 6] we compare the predictions of the AdS/CFT inspired model with the $F_{2}^{D(3)}$ ZEUS data for different values of $\beta$. For comparison, we also present the RC BK prediction, which was recently analyzed in detail in the Ref. [78] and a good description of the data in a large $Q^{2}$ range was found. The additional parameters in our calculations using the AdS/CFT inspired model are the strong coupling constant, which determines the normalization of the $q \bar{q} g$ contribution, and the diffractive slope $B_{D}$. Following Ref. [78] we assume $\alpha_{s}=0.15$ and constrain the diffractive slope $B_{D}$ by the value of $\sigma_{0}$ through $\sigma_{0}=4 \pi B_{D}$ assuming a Gaussian form factor for the proton [54]. As already demonstrated in [78] the RC BK solution describes reasonably well the data. In contrast, the AdS/CFT inspired predictions underestimate the ZEUS data, which is in the kinematical range $x_{I P} \gtrsim 10^{-4}$. For $10^{-7} \lesssim x_{I P} \lesssim 10^{-4}$ is hard to distinguish between the parametrizations. However, for $x_{I P} \lesssim 10^{-7}$ and all $\beta$ the AdS/CFT inspired model predict that the diffractive structure function saturates, while the RC BK solution predicts a steep growth at small $x_{I P}$. This behavior is directly related to the energy behavior of the dipole scattering amplitude shown in Figs. 1 and 2 At $x_{I P}=10^{-8}$ the predictions differ by a factor 1.5.

Finally, in Fig. 7 we present the energy dependence of the ratio $R_{\sigma}=\frac{\sigma_{t o t}^{D}}{\sigma_{t o t}}$, which has been considered an important signature of the saturation physics [49, 81$]$. In the range $10^{-7} \lesssim x \lesssim 10^{-4}$ both models predict a similar behavior. At 


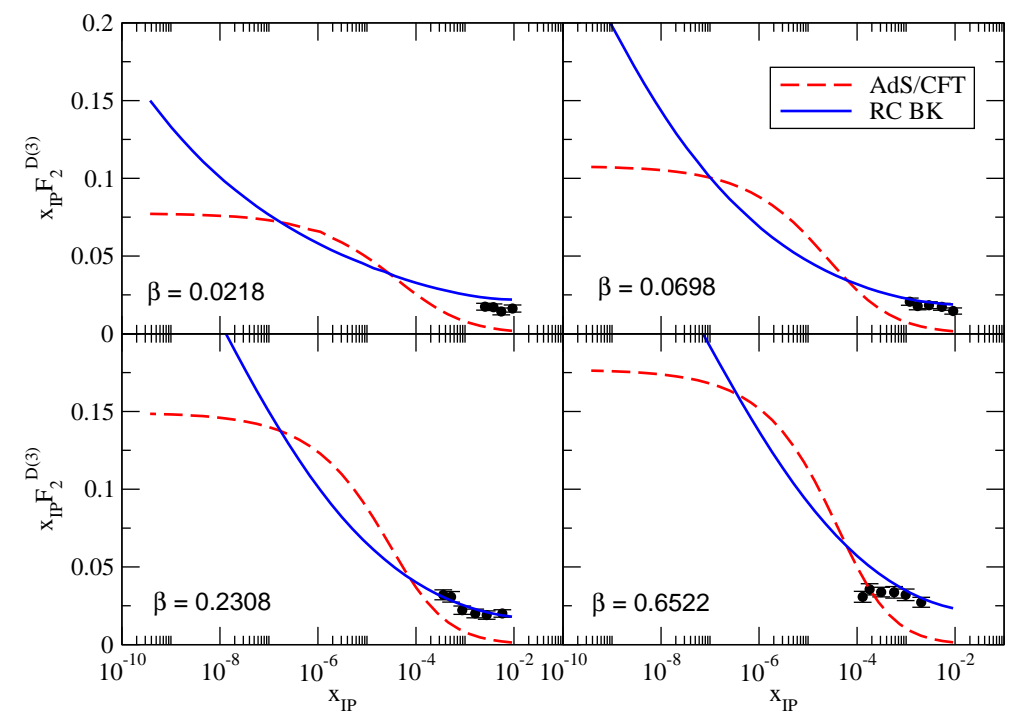

FIG. 6: Predictions for $F_{2}^{D(3)}\left(Q^{2}, \beta, x_{\mathbb{P}}\right)$ compared with the ZEUS data [80]. Solid line: RC BK model; Dashed line: AdS/CFT model.

$x_{I P} \lesssim 10^{-7}$ the models predicts distincts behaviours, being $R_{\sigma}$ energy independent for $x \lesssim 10^{-7}$ from the AdS/CFT model. This behavior is directly associated to the $x$ dependence of the dipole amplitude, predicted by the models (see Fig. 2). Therefore, only at $x \lesssim 10^{-7}$ one expects to find some distinction between the parametrizations for this observable, which means that only at very small $x$ we expect some difference between perturbative and strong coupling models.

\section{CONCLUSIONS}

Disentangle the hard and soft dynamics in diffractive DIS is one of the main open questions of the strong interactions. Although Regge theory is able to reproduce the experimental data for hadronic collisions, the understanding of this theory in terms of QCD is still lacking, which is directly associated to the fact that strong coupling analytical calculations are not possible in QCD. It is expected that considering the high energy scattering amplitude in $N=4$ SYM theory using the AdS/CFT correspondence one can guess which physics phenomena could be important in the strong coupling limit of QCD. In this paper we have investigated inclusive and diffractive lepton-hadron DIS, within the dipole formalism, through a parametrization for the dipole-hadron scattering amplitude presented recently in [33], inspired on the AdS/CFT correspondence, proposed to describe the evolution and scattering at small values of $x$ and $Q^{2}$, i.e., proposed to describe a nonperturbative domain, characterized by large values of the coupling constant. We have compared the results with a perturbative QCD-based framework where the scattering amplitude is the solution of the nonlinear BK evolution equation with running coupling effects [42]. Both analyses are similar only in a restrict kinematical range $\left(10^{-7} \lesssim x \lesssim 10^{-4}\right)$, and become quite different when $x$ goes towards large (where the AdS/CFT dipole model is not valid) and extremely small values, which are not experimentaly available yet. In particular, the diffractive structure function $F_{2}^{D(3)}$, which has been shown to be well described by the solution of the running coupling BK equation [78], is underestimated by the analysis using the AdS/CFT dipole model in the restricted range of available experimental data. Our results indicate that the strict AdS/CFT approach behind the dipole model proposed in [33], could not be the right approach for the treatment of diffractive deep inelastic scattering in the HERA kinematical range. Moreover, discriminating between the perturbative RC BK approach and the nonperturbative AdS/CFT inspired approach in the kinematical range of the future $e p$ colliders [79] will be a hard task.

The noticeable difference between RC BK and AdS/CFT analyses at extremely small values of $x$ is due to the asymptotic prediction of the latter, i.e., the saturation scale saturates in the $x \rightarrow 0$ limit, and it is a characteristic of the particular AdS/CFT model derived in [33]. It must be pointed out, that, besides surprising, this behavior of $Q_{s}$ 


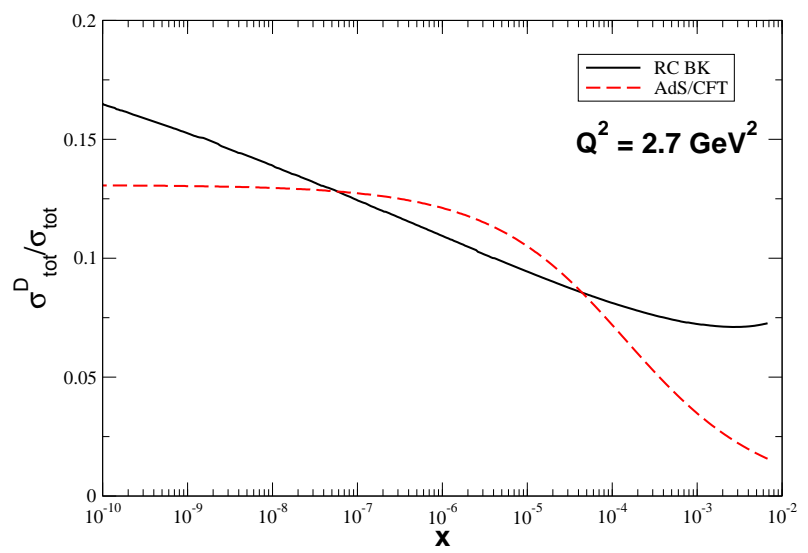

FIG. 7: Prediction for the $x$ dependence of the ratio $\sigma_{\text {tot }}^{D} / \sigma_{t o t}$.

is also controversial, as it was argued in 31]. In particular, it is in sharp contradiction with all other calculations of the scattering amplitudes in the context of the AdS/CFT correspondence, whose predictions give $Q_{s}^{2} \sim 1 / x$, i.e., the saturation scale grows much faster than the corresponding scale in perturbative QCD. An energy-dependent saturation scale appears to be consistent with the high energy dynamics at strong coupling and, as it was demonstrated in [31], it is necessary to ensure energy-momentum conservation. As discussed in the Introduction, the basic difference between the model proposed in [33] and the other ones in the literature is the nature of the projectile, which is assumed to be a color dipole in [33], instead of a virtual photon as in [31]. This difference should be unimportant at a fundamental level, since the property of unitarization refers to the mechanism of inelastic scattering. It implies that, probably, the different energy dependence of the saturation scale should be associated to some subtle mathematical manipulation which were not fully under control. Finally, if the behavior $Q_{s}^{2} \sim 1 / x$ is the correct one, it can be expected that the resulting AdS/CFT predictions will be closer to the perturbative BK predictions in the kinematical range of the future colliders. Therefore, in order to discriminate between the different formalisms, it will be necessary to search other less inclusive observables, as for example, exclusive vector meson photoproduction.

\section{Acknowledgments}

This work was partially financed by the Brazilian funding agencies CNPq, CAPES and FAPERGS.

[1] E. Iancu and R. Venugopalan, arXiv:hep-ph/0303204; H. Weigert, Prog. Part. Nucl. Phys. 55, 461 (2005); E. Iancu, Nucl. Phys. Proc. Suppl. 191, 281 (2009).

[2] V.N. Gribov and L.N. Lipatov, Sov. J. Nucl. Phys. 15, 438 (1972); G. Altarelli and G. Parisi, Nucl. Phys. B126, 298 (1977); Yu.L. Dokshitzer, Sov. Phys. JETP 46, 641 (1977).

[3] CTEQ Collaboration. Rev. Mod. Phys. 67, 157 (1995).

[4] A. H. Mueller. Phys. Lett. B396, 251 (1997).

[5] E. Iancu, A. Leonidov and L. McLerran, Nucl.Phys. A692, 583 (2001).

[6] E. Ferreiro, E. Iancu, A. Leonidov and L. McLerran, Nucl. Phys. A701, 489 (2002).

[7] J. Jalilian-Marian, A. Kovner, L. McLerran and H. Weigert, Phys. Rev. D 55, 5414 (1997).

[8] J. Jalilian-Marian, A. Kovner and H. Weigert, Phys. Rev. D 59, 014014 (1999).

[9] J. Jalilian-Marian, A. Kovner and H. Weigert, Phys. Rev. D59, 014015 (1999).

[10] J. Jalilian-Marian, A. Kovner and H. Weigert, Phys. Rev. D 59, 034007 (1999).

[11] A. Kovner, J. Guilherme Milhano and H. Weigert, Phys. Rev. D 62, 114005 (2000).

[12] H. Weigert, Nucl. Phys. A703, 823 (2002).

[13] I. I. Balitsky, Nucl. Phys. B463, 99 (1996).

[14] I. I. Balitsky, Phys. Rev. Lett. 81, 2024 (1998). 
[15] I. I. Balitsky, Phys. Rev. D 60, 014020 (1999).

[16] I. I. Balitsky, Phys. Lett. B 518, 235 (2001).

[17] I.I. Balitsky and A.V. Belitsky, Nucl. Phys. B629, 290 (2002).

[18] Y.V. Kovchegov, Phys. Rev. D 60, 034008 (1999).

[19] Y.V. Kovchegov, Phys. Rev. D 61, 074018 (2000).

[20] J. M. Maldacena, Adv. Theor. Math. Phys. 2, 231 (1998) [Int. J. Theor. Phys. 38, 1113 (1999) ].

[21] S. S. Gubser, I. R. Klebanov and A. M. Polyakov, Phys. Lett. B 428, 105 (1998).

[22] E. Witten, Adv. Theor. Math. Phys. 2, 253 (1998).

[23] E. Iancu, Acta Phys. Polon. B 39, 3213 (2008).

[24] J. Polchinski and M. J. Strassler, Phys. Rev. Lett. 88, 031601 (2002) ; JHEP 0305, 012 (2003).

[25] L. Cornalba and M. S. Costa, Phys. Rev. D 78, 096010 (2008); L. Cornalba, M. S. Costa and J. Penedones, arXiv:0911.0043 [hep-th]; arXiv:1001.1157 [hep-ph].

[26] C. A. Ballon Bayona, H. Boschi-Filho and N. R. F. Braga, JHEP 0803, 064 (2008); JHEP 0810, 088 (2008); JHEP 0809, 114 (2008); arXiv:0912.0231 [hep-th].

[27] Y. Hatta, E. Iancu and A. H. Mueller, JHEP 0801, 026 (2008).

[28] Y. Hatta, E. Iancu and A. H. Mueller, JHEP 0801, 063 (2008).

[29] E. Levin, J. Miller, B. Z. Kopeliovich and I. Schmidt, JHEP 0902, 048 (2009).

[30] B. Pire, C. Roiesnel, L. Szymanowski and S. Wallon, Phys. Lett. B 670, 84 (2008).

[31] E. Avsar, E. Iancu, L. McLerran and D. N. Triantafyllopoulos, JHEP 0911, 105 (2009).

[32] B. Hassanain and M. Schvellinger, arXiv:0912.4704 [hep-th].

[33] J. L. Albacete, Y. V. Kovchegov and A. Taliotis, JHEP 0807, 074 (2008).

[34] R. A. Janik and R. Peschanski, Phys. Rev. D 73, 045013 (2006).

[35] Y. V. Kovchegov, Z. Lu and A. H. Rezaeian, Phys. Rev. D 80, 074023 (2009).

[36] M. B. Gay Ducati and V. P. Goncalves, Phys. Lett. B 502, 92 (2001).

[37] Y. V. Kovchegov and H. Weigert, Nucl. Phys. A 784, 188 (2007).

[38] J. L. Albacete and Y. V. Kovchegov, Phys. Rev. D 75, 125021 (2007).

[39] I. Balitsky, Phys. Rev. D 75, 014001 (2007); I. Balitsky and G. A. Chirilli, Phys. Rev. D 77, 014019 (2008).

[40] Y. V. Kovchegov and H. Weigert, Nucl. Phys. A 789, 260 (2007).

[41] J. L. Albacete, Phys. Rev. Lett. 99, 262301 (2007).

[42] J. L. Albacete, N. Armesto, J. G. Milhano and C. A. Salgado, Phys. Rev. D80, 034031 (2009).

[43] H. Weigert, J. Kuokkanen and K. Rummukainen, AIP Conf. Proc. 1105, 394 (2009).

[44] A.H. Mueller. Eur. Phys. J. A1, 19 (1999).

[45] A. Hebecker. Phys. Rept. 331, 1 (2000).

[46] N. N. Nikolaev and B. G. Zakharov, Z. Phys. C49, 607 (1991); Z. Phys. C53, 331 (1992).

[47] V. Barone and E. Predazzi, High-Energy Particle Diffraction, Springer-Verlag, Berlin Heidelberg, (2002).

[48] K. Golec-Biernat and M. Wusthoff, Phys. Rev. D 59, 014017 (1999).

[49] K. Golec-Biernat and M. Wusthoff, Phys. Rev. D 60, 114023 (1999).

[50] M. Wusthoff, Phys. Rev. D 56, 4311 (1997).

[51] J. R. Forshaw, R. Sandapen and G. Shaw, Phys. Lett. B594, 283 (2004).

[52] N. N. Nikolaev and B. G. Zakharov, J. Exp. Theor. Phys. 78, 598 (1994) [Zh. Eksp. Teor. Fiz. 105,1117 (1994) ]; Z. Phys. C64, 631 (1994); N. N. Nikolaev, W. Schaefer, B. G. Zakharov and V. R. Zoller, JETP Lett. 80, 371 (2004) [Pisma Zh. Eksp. Teor. Fiz. 80, 423 (2004)].

[53] K. Golec-Biernat and A. Luszczak, Phys. Rev. D 79, 114010 (2009).

[54] C. Marquet, Phys. Rev. D 76, 094017 (2007).

[55] J. Bartels, K. Golec-Biernat, H. Kowalski, Phys. Rev. D 66, 014001 (2002).

[56] H. Kowalski and D. Teaney, Phys. Rev. D 68, 114005 (2003).

[57] H. Kowalski, L. Motyka and G. Watt, Phys. Rev. D 74, 074016 (2006).

[58] K. Golec-Biernat and S. Sapeta, Phys. Rev. D 74, 054032 (2006).

[59] J. T. de Santana Amaral, M. B. Gay Ducati, M. A. Betemps and G. Soyez, Phys. Rev. D 76, 094018 (2007).

[60] C. Marquet, R. Peschanski and G. Soyez, Phys. Rev. D 76, 034011 (2007).

[61] G. Soyez, Phys. Lett. B 655, 32 (2007).

[62] G. Watt and H. Kowalski, Phys. Rev. D 78, 014016 (2008).

[63] E. Iancu, K. Itakura, S. Munier, Phys. Lett. B590, 199 (2004).

[64] D. Kharzeev, Y.V. Kovchegov and K. Tuchin, Phys. Lett. B599, 23 (2004).

[65] A. Dumitru, A. Hayashigaki and J. Jalilian-Marian, Nucl. Phys. A 765, 464 (2006).

[66] A. Dumitru, A. Hayashigaki and J. Jalilian-Marian, Nucl. Phys. A 770, 57 (2006).

[67] V. P. Goncalves, M. S. Kugeratski, M. V. T. Machado and F. S. Navarra, Phys. Lett. B 643, 273 (2006).

[68] D. Boer, A. Utermann, E. Wessels, Phys. Rev. D 77, 054014 (2008).

[69] J. R. Forshaw, G. Kerley and G. Shaw, Phys. Rev. D 60, 074012 (1999).

[70] L. N. Lipatov, Sov. J. Nucl. Phys. 23, 338 (1976); E. A. Kuraev, L. N. Lipatov and V. S. Fadin, Sov. Phys. JETP 45, 199 (1977); I. I. Balitsky and L. N. Lipatov, Sov. J. Nucl. Phys. 28, 822 (1978).

[71] E. Iancu, K. Itakura and L. McLerran, Nucl. Phys A708, 327 (2002).

[72] A. H. Mueller and D. N. Triantafyllopoulos, Nucl. Phys. B640, 331 (2002).

[73] N. Armesto and M. A. Braun, Eur. Phys. J. C20, 517 (2001). 
[74] M. A. Braun, Phys. Lett. B 576, 115 (2003).

[75] J. L. Albacete, N. Armesto, J. G. Milhano, C. A. Salgado, and U. A. Wiedemann, Phys. Rev. D 71, 014003 (2005).

[76] http://www-fp.usc.es/phenom/rcbk.

77] M. S. Kugeratski, V. P. Goncalves and F. S. Navarra, Eur. Phys. J. C 44, 577 (2005).

[78] M. A. Betemps, V. P. Goncalves and J. T. de Santana Amaral, Eur. Phys. J. C 66, 137 (2010).

[79] P. Newman, Nucl. Phys. Proc. Suppl. 191, 307 (2009).

[80] S. Chekanov et al. [ZEUS Collaboration], Nucl. Phys. B 713, 3 (2005); Nucl. Phys. B 800, 1 (2008).

[81] M. S. Kugeratski, V. P. Goncalves and F. S. Navarra, Eur. Phys. J. C 46, 412 (2006). 